Artículo

\title{
Caracterización de la respuesta a la sequía de líneas segregantes de maíz
}

\author{
Elizabeth Ibarra Sánchez 1 \\ Antonio Castillo Gutiérrez ${ }^{2 \S}$ \\ María Eugenia Núñez Valdéz ${ }^{3}$ \\ Ramón Suárez Rodríguez ${ }^{4}$ \\ María Andrade Rodríguez ${ }^{1}$ \\ Francisco Perdomo Roldán ${ }^{2}$ \\ ${ }^{1}$ Facultad de Ciencias Agropecuarias-Universidad Autónoma del Estado de Morelos. Av. Universidad núm. \\ 1001, Col. Chamilpa, Cuernavaca, Morelos. CP. 62209. (Ibarra_lizanz@hotmail.com; \\ maria.andrade@uaem.mx). ${ }^{2}$ Escuela de Estudios Superiores de Xalostoc-UAEM. \\ (fperdomor@yahoo.com.mx). $\quad{ }^{3}$ Centro de Investigación en Dinámica Celular-UAEM. \\ (eugenia@buzon.uaem.mx). ${ }^{4}$ Centro de Investigación en Biotecnología-UAEM. (rsuarez@uaem.mx).
}

${ }^{\S}$ Autor para correspondencia: antonio.castillo@uaem.mx.

\section{Resumen}

El estrés por sequía es una de las principales causas de la reducción del rendimiento del maíz en los trópicos, principalmente en la agricultura que depende de lluvias, por lo que se requiere generar genotipos con tolerancia a sequía. Los objetivos de la investigación fueron cuantificar la respuesta agronómica de un grupo de líneas $\mathrm{S}_{2}$ segregantes de maíz en ambientes de riego y sequía, así como seleccionar líneas con tolerancia al déficit hídrico, con base en dos índices y al promedio de rendimiento de ambos ambientes. Los genotipos de estudio fueron 96 líneas $\mathrm{S}_{2}$ segregantes, dos líneas testigo y dos líneas no seleccionadas para sequía. Las líneas se evaluaron bajo riego y sequía inducida usando un diseño Látice Alfa con cuatro repeticiones, en Ayala, Morelos, México. Se midieron 10 variables morfológicas y se estimaron los índices de susceptibilidad a la sequía (ISS) y el índice de tolerancia (IT). Con base en el rendimiento de grano en riego (3.14 t ha $\left.{ }^{-1}\right)$, se observó una reducción de $77.7 \%$ del rendimiento en sequía $\left(0.7 \mathrm{t} \mathrm{ha}^{-1}\right)$. Los índices ISS e IT identificaron a las líneas LUM69, LUM82 y LUM30 como tolerantes a sequía, solo LUM69 mostró un rendimiento competitivo en ambos ambientes. Los índices de ISS e IT como criterio para identificar genotipos tolerantes, no seleccionaron a las líneas con mejor rendimiento de grano bajo condiciones de riego, por lo que se debe incluir en los criterios de selección al promedio de rendimiento a través de los ambientes de humedad.

Palabras clave: índices de susceptibilidad, líneas $\mathrm{S}_{2}$, tolerancia a la sequía.

Recibido: mayo de 2020

Aceptado: julio de 2020 


\section{Introducción}

El maíz (Zea mays L.), es de mayor importancia en México, de manera que en 2018 se establecieron aproximadamente 7.4 millones de hectáreas, con un rendimiento promedio de $3.8 \mathrm{tha}^{-1}$; donde $72 \%$ se estableció en temporal y el resto en condiciones de riego. En el estado de Morelos se siembran cerca de 38 mil hectáreas de maíz, de las cuales 93\% se realiza en condiciones de precipitación pluvial, con un promedio de rendimiento de grano de $4 \mathrm{t} \mathrm{ha}^{-1}$ (SIAP-SAGARPA, 2019).

En México, 52\% de su superficie agrícola se cataloga como zona árida o semiárida, lo que la hace altamente susceptible a sequía (Salinas et al., 1998). El déficit hídrico ocurre cuando la cantidad de lluvia ha sido significativamente más baja de lo normal, respecto al índice anual de precipitación. Por otra parte, los efectos del estrés por falta de agua se relacionan directamente con la intensidad y duración del déficit hídrico, lo que provoca una reducción significativa en el rendimiento de grano (CONAZA, 2010; FAO-SAGARPA, 2012; Esparza, 2014).

La sequía, es el factor más importante que limita la productividad del maíz en regiones que dependen de las lluvias (Srivatava, 2014; Shafiq et al., 2015). En México, en los últimos 14 años se han reportado altos niveles de sequía en el ciclo agrícola primavera-verano; particularmente en el año 2011, se registró déficit de lluvias durante los meses de junio a septiembre, afectando 213\% de la superficie sembrada con maíz para grano (SMN, 2017; SIAP-SAGARPA, 2017).

La magnitud del efecto del déficit hídrico en el rendimiento de grano de maíz, dependen de la intensidad, duración, y etapa de fenológica en que se presenta, cuando la sequía ocurre en la fase vegetativa, se reduce la altura de planta y la duración del área foliar, lo que afecta la tasa fotosintética; sin embargo, el periodo más sensible en la planta de maíz es el periodo constituido entre una semana antes y dos semanas después de floración masculina (Stone et al., 2001; Avendaño et al., 2005).

El efecto mayor observado es el retraso de la floración femenina, incrementando el intervalo antesis-emergencia de estigmas, lo que reduce la producción final de grano (Westgate y Boyer, 1986; Saini y Westgate, 2000). Cundo la sequía es prologada se afecta el crecimiento, desarrollo y la producción en la planta de maíz, como una consecuencia del impacto en procesos fisiológicos y bioquímicos (Khalili et al., 2013).

En la cuantificación del efecto de la sequía en plantas cultivadas, se han desarrollado índices que permiten medir la respuesta de genotipos al déficit hídrico, la mayoría de los índices se calculan con base en el rendimiento de grano en condiciones de riego y sequía (Fischer y Maurer, 1978; Rosielle y Hamblin, 1981), lo que hace necesario identificar características agronómicas relacionadas con la adaptación a condiciones limitantes de humedad, lo que facilita la selección de genotipos sobresalientes en forma práctica y eficiente.

El fitomejoramiento convencional ha desarrollado genotipos con tolerancia a la sequía, por medio de métodos que evalúan y seleccionan genotipos en condiciones limitantes y no limitantes de humedad, lo que ha permitido identificar características deseables que faciliten obtener la mayor ganancia genética en ambas condiciones de humedad (Muñoz, 1980; Fischer et al., 1989; Bruce et al., 2002). 
Los criterios de selección más usados para tolerancia a sequía, se enfocan en el rendimiento de grano y caracteres secundarios que contribuyen a optimizar la respuesta de las plantas al déficit de agua (Badu-Apraku et al., 2011), los caracteres secundarios comúnmente usados en la selección indirecta para tolerancia a sequía son, corto intervalo antesis-emergencia de estigmas, baja senescencia foliar, espiga pequeña y hojas arriba de la mozorca erectas (Edmeades et al., 1999), debido a que la selección directa para rendimiento de grano en ambientes limitantes de humedad es complicado, por la baja heredabilidad de éste carácter (Ribaut et al., 1996).

Un método eficiente para incrementar la tolerancia y estabilizar el rendimiento en condiciones de baja humedad, es la selección recurrente de genotipos de alto rendimiento a través de ambientes con déficit y sin déficit de humedad. Este método ha demostrado ser efectivo en maíz; sin embargo, es costoso y tardado, en la población de maíz Tuxpeño Sequía obtenida a través de ocho ciclos de selección bajo déficit de agua, se incrementó el rendimiento de grano en $108 \mathrm{~kg} \mathrm{ha}^{-1}$ por ciclo (Bolaños y Edmeades, 1993). En tanto que, en otro estudio, se reportó que la selección recurrente de cinco ciclos de selección continua, el rendimiento de grano en sequía tuvo una ganancia genética de $12 \%$ por ciclo de selección (Chapman y Edmeades, 1999).

La formación e identificación de líneas endogámicas segregantes a partir de la generación $\mathrm{F}_{2}$, potencializa la generación de varianza genética por la segregación alélica originada por el cruzamiento de líneas altamente endogámicas contrastantes en el carácter de interés (Walsh, 2001). A partir de la cruza entre las líneas SD34 y SD35 contrastantes en tolerancia a sequía, se derivaron 230 familias $\mathrm{F}_{3}$, detectando correlaciones fenotípicas entre el rendimiento de grano y el intervalo antesis-emergencia de estigmas $\left(-0.5^{* *}\right)$, así como con el número de mazorcas por planta $\left(0.53^{* *}\right)$, las heredabilidades relativamente altas en sequía en estas características, facilitó la selección indirecta de genotipos de alto rendimiento en sequía (Agrama y Moussa, 1996).

La línea progenitora tolerante del presente estudio (Ac7643), se usó también como progenitor tolerante para generar 234 familias segregantes, las que se evaluaron en condiciones de sequía intermedia y severa, lo mismo que en riego, las variables de floración masculina, femenina, intervalo antesis-emergencia de estigmas (ASI) y rendimiento de grano manifestaron segregación transgresiva en sequía severa, el ASI de las familias mostró un rango de -4.4 a 9.3 días y en el rendimiento de grano se observó una reducción de $61 \%$ del rendimiento en sequía; en cuanto al comportamiento de Ac7643 se reportó un ASI corto de -0.2 días en estrés intermedio y de -0.6 días bajo estrés hídrico severo, con un rendimiento de grano $1326 \mathrm{~kg}$. ha ${ }^{-1}$ en el ambiente de sequía (Ribaut et al., 1996 y 1997). El comportamiento de la línea progenitora susceptible B39 en ambientes de contrastantes de humedad, presentó en riego valores del intervalo antesis-emergencia de estigmas y rendimiento de grano de $1.9 \mathrm{~d} \mathrm{y} 2.4 \mathrm{t} \mathrm{ha}^{-1}$, respectivamente; en tanto que, en sequía los valores para las mismas variables fueron de $9.8 \mathrm{~d}$ y de $0.4 \mathrm{t} \mathrm{ha}^{-1}$ (Castillo, 2004).

Uno de los propósitos de generar poblaciones segregantes a partir del cruzamiento entre una línea tolerante y una susceptible, es generar nueva variación genética y tener la posibilidad de detectar herencia transgresiva en la expresión fenotípica de características de interés. Los objetivos de la investigación fueron cuantificar la respuesta agronómica de un grupo de líneas $\mathrm{S}_{2}$ segregantes de maíz en ambientes de riego y sequía, así como seleccionar líneas con tolerancia al déficit hídrico, con base al promedio de rendimiento de ambos ambientes. 


\section{Materiales y métodos}

El germoplasma de estudio estuvo constituido por 100 genotipos de maíz, de los cuales 96 fueron líneas $\mathrm{S}_{2}$ segregantes, la línea testigo T-43 con tolerancia a la sequía, línea testigo Ac7729 susceptible a la sequía y dos líneas $S_{2}$, provenientes de otra población segregante. Las líneas $S_{2}$ provienen, del cruzamiento de las líneas Ac7643 (tolerante a sequía) y B39 (susceptible a sequía). Las líneas endogámicas T-43 y B39 fueron proporcionados por el Instituto Nacional de Investigaciones Forestales Agrícolas y Pecuarias (INIFAP), en tanto que las líneas Ac7643 y Ac7729 fueron facilitadas por el Centro Internacional de Mejoramiento de Maíz y Trigo (CIMMYT).

Las líneas se evaluaron en experimentos de campo en condiciones de riego y sequía inducida; se establecieron en el campo experimental de la Escuela de Estudios Superiores de Xalostoc, de la Universidad Autónoma del Estado de Morelos, en el municipio de Ayala, Morelos. La localidad se caracteriza por presentar un clima cálido Sub-húmedo, suelo tipo vertisol, a $1285 \mathrm{~m}$ de altitud con rango de temperaturas de 8.4 a $36{ }^{\circ} \mathrm{C}$ y una precipitación media anual de $912 \mathrm{~mm}$ (INEGI, 2008).

El ambiente de riego se estableció en el ciclo de lluvias correspondiente a primavera-verano 2016, período en el cual no hubo limitación de humedad para el cultivo, registrándose $1089 \mathrm{~mm}$ de precipitación total durante el ciclo biológico del cultivo. En cuanto al ambiente de sequía, se estableció en el ciclo agrícola otoño-invierno 2016-2017 (donde comúnmente no se presentan lluvias), para el manejo de humedad en éste ambiente, suministró un riego de siembra (90 mm), uno de emergencia $(40 \mathrm{~mm})$ a los 9 días después del de siembra, posteriormente tres riegos de auxilio de $55 \mathrm{~mm}$ cada uno, a intervalos de $12 \mathrm{~d}$, suspendiéndose la irrigación a los $45 \mathrm{~d}$ después de la siembra; a los 77 d se suministró un riego ligero $(30 \mathrm{~mm})$ aplicando el riego en surcos alternos y a partir del día 94 se proporcionaron dos riegos de $30 \mathrm{~mm}$ cada uno, para favorecer el llenado de grano, aplicándose una lámina total de riego de $385 \mathrm{~mm}$.

El diseño experimental utilizado en ambos ambientes fue un Látice Alfa $(0,1)$, con cuatro repeticiones por entrada y por ambiente; los genotipos se sembraron en 10 bloques, con 10 unidades experimentales por bloque. La unidad experimental fue un surco de $4 \mathrm{~m}$ de largo, $0.75 \mathrm{~m}$ de ancho y a $0.2 \mathrm{~m}$ la distancia entre plantas. Los manejos agronómicos de los experimentos de campo fueron conducidos de acuerdo con la guía técnica del Campo Experimental de Zacatepec, Morelos (Trujillo, 2002). Se registraron 10 variables de respuesta, floración masculina y femenina (FM-d y FF-d), intervalo antesis emergencia de estigmas (ASI-d), las variables relacionadas con la floración se registraron en la etapa fenológica VT y R1, altura de planta y mazorca (AP-cm y AM-cm), en cuanto a las alturas se midieron en la etapa R4, hileras por mazorca (HM-No.), longitud de mazorca $(\mathrm{LM}-\mathrm{cm})$ diámetro de mazorca $(\mathrm{DM}-\mathrm{cm})$, peso de 100 granos $(\mathrm{P} 100 \mathrm{G}-\mathrm{g})$ y rendimiento de grano $\left(\mathrm{RG}_{\mathrm{t}} \mathrm{ha}^{-1}\right)$, la ultimas variables se determinaron posterior a la madurez comercial.

Los datos generados por los experimentos se sometieron a análisis de varianza combinados a través de los dos ambientes de evaluación (riego-sequía) para las 10 variables de respuesta, posteriormente se realizo una comparación múltiple de medias mediante el procedimiento de diferencia mínima significativa $(\alpha=0.05)$ y un análisis de regresión lineal. 
Con el propósito de cuantificar la respuesta de las líneas a la sequía, se determinaron el índice de susceptibilidad a la sequía (ISS) [Fischer y Maurer, 1978] y el índice de tolerancia (IT) [Rosielle y Hamblin, 1981]. El ISS se estimó por la ecuación siguiente: ISS=1-[Ys/Yr]/1 - [Ysm/Yr]. Donde: Ys= promedio de rendimiento de la i-ésima línea en el ambiente de sequía; Yr= promedio de rendimiento de la i-ésima línea en el ambiente de riego; Ysm= promedio de rendimiento de todas las líneas en el ambiente de sequía y Yrm= promedio de rendimiento de todas las líneas en el ambiente de riego. En el ISS los valores menores a uno indican relativa tolerancia a la sequía en los genotipos.

En cuanto al IT se calculó mediante la ecuación: $\mathrm{IT}=\mathrm{Y}_{1}-\mathrm{Y}_{2}$. Donde: $\mathrm{Y}_{1}=$ el rendimiento de grano de la línea en el ambiente de riego, $\mathrm{Y}_{2}=$ rendimiento de grano de la línea en el ambiente de sequía. En el IT los valores relativamente más bajos, indican que un genotipo es tolerante a la sequía.

Los valores de ambos índices se calcularon para cada genotipo y cada repetición, lo que permitió realizar un análisis de varianza de un vía, para posteriormente realizar una prueba de comparación de medias, por medio de una prueba $\mathrm{DMS}_{(0.05)}$. La totalidad de los análisis estadísticos se realizaron usando el software Statistical Analysis System (SAS, 2009).

\section{Resultados y discusión}

Las líneas en el ambiente de riego produjeron un promedio del rendimiento de grano de $3.14 \mathrm{t}$ $\mathrm{ha}^{-1}$, en comparación al promedio del ambiente de sequía de $0.7 \mathrm{t} \mathrm{ha}^{-1}$, lo que indujo una reducción de $77.7 \%$ por causa de la sequía en combinación con altas temperaturas; lo que se sustenta en los $1089 \mathrm{~mm}$ de precipitación registrada en el ambiente de riego, con temperaturas fluctuando de 27.7 a $29.5{ }^{\circ} \mathrm{C}$, en tanto que en sequía, se aplicó una lámina total de riego de $385 \mathrm{~mm}$ y se registraron temperaturas de 28.8 a $33.9{ }^{\circ} \mathrm{C}$, restringiéndose la humedad cerca de la floración masculina.

El análisis de varianza combinado (Cuadro 1) en las fuentes de variación de Ambientes, Líneas y la interacción Línea x Ambiente mostró diferencias altamente significativas $(p \leq 0.01)$ para todas las variables estudiadas. Las diferencias estadísticas entre ambientes (riego-sequía) detectadas por el Anova combinado, se explican por el contrastante en las condiciones de humedad a la cual se sometieron los genotipos y por los ciclos agrícolas de evaluación; es decir, las condiciones ambientales como temperatura y humedad relativa que prevalecieron en el ciclo agrícola primavera-verano fluctuaron entre $15.7^{\circ} \mathrm{C}$ y $28.3{ }^{\circ} \mathrm{C}, 74.7$ a $78.4 \%$, respectivamente.

En tanto que, en el ciclo otoño-invierno la temperatura osciló entre $13.3{ }^{\circ} \mathrm{C}$ y $32.4^{\circ} \mathrm{C}$ y la humedad relativa entre 31.3 a 51\% (INIFAP, 2017; SMN, 2017). La variación en la temperatura del aire y humedad relativa entre los ciclos agrícolas de evaluación, tienen efecto directo en el grado de expresión fenotípica, particularmente en variables morfológicas, por lo que, las fluctuaciones ambientales tienen un impacto en la respuesta agronómica del germoplasma a través de diferentes sitios y ambientes de prueba (Zambrano et al., 2017).

En cuanto, a las diferencias estadísticas detectadas entre líneas, esta se atribuye a la variación genética presente en el grupo de líneas evaluado, debido a que son líneas segregantes provenientes del cruzamiento de dos líneas contrastantes en respuesta a la sequía, lo que generó 
segregación alélica en múltiples loci lo que se reflejó en una amplia variación en la respuesta agronómica de las líneas. Por otro lado, la selección directa para rendimiento de grano bajo sequía, ha sido considerada ineficiente debido a la baja heredabilidad del carácter (Ribaut et al., 1996), por lo que se considera más efectiva la selección de líneas tolerantes, el evaluarlas en condiciones limitantes y no limitantes de agua.

Cuadro 1. Valores estadísticos de características morfológicas de líneas $\mathrm{S}_{2}$ de maíz (Zea mays $\mathbf{L}$.) evaluadas bajo riego-sequía en Ayala, Morelos, México.

\begin{tabular}{cccccccc}
\hline FV & Amb & Reps/Amb & Bl/Reps Amb & Líneas & L x Amb & Error & CV (\%) \\
\hline GL & 1 & 6 & 72 & 99 & 99 & 522 & \\
FM d & $73594^{* *}$ & $9.72^{* *}$ & $1.4^{*}$ & $12.99^{* *}$ & $5.07^{* *}$ & 1.03 & 1.41 \\
FF d & $93723^{* *}$ & $18.84^{* *}$ & $3.52^{* *}$ & $16.94^{* *}$ & $8.33^{* *}$ & 2.25 & 2.01 \\
ASI & $2.1^{* *}$ & $0.01 \mathrm{~ns}$ & $0.01 \mathrm{~ns}$ & $0.01^{* *}$ & $0.01^{* *}$ & 0.01 & 4.02 \\
AP cm & $3674313^{* *}$ & $1556.27^{* *}$ & $393.81^{* *}$ & $1061.67^{* *}$ & $471.8^{* *}$ & 84.82 & 5.19 \\
AM cm & $1070777^{* *}$ & $314.46^{* *}$ & $161.06^{* *}$ & $834.1^{* *}$ & $291.36^{* *}$ & 70.28 & 10.05 \\
HM No. & $1682^{* *}$ & $14.79^{* *}$ & $1.68^{*}$ & $14.19^{* *}$ & $4.19^{* *}$ & 1.2 & 8.34 \\
LM cm & $1212.54^{* *}$ & $5.19 \mathrm{~ns}$ & $2.44^{* *}$ & $7.02^{* *}$ & $3.61^{* *}$ & 1.82 & 11.7 \\
DM cm & $147.49^{* *}$ & $0.66^{*}$ & $0.06 \mathrm{~ns}$ & $0.32^{* *}$ & $0.08^{* *}$ & 0.05 & 6.73 \\
P100G g & $8719.53^{* *}$ & $13.41^{*}$ & $4.34 \mathrm{~ns}$ & $32.19^{* *}$ & $8.42^{* *}$ & 3.36 & 10.98 \\
RG t ha & $1201.86^{* *}$ & $1.29^{*}$ & $0.43 \mathrm{~ns}$ & $2.6^{* *}$ & $1.6^{*}$ & 0.4 & 32.61 \\
\hline
\end{tabular}

$\mathrm{FV}=$ fuente de variación; $\mathrm{GL}=$ grados de libertad; $\mathrm{ns}=$ no significativo; ${ }^{*}=$ significativo al $5 \% ;{ }^{* *}=$ significativo al $1 \%$; $\mathrm{FM}=$ floración masculina; $\mathrm{FF}=$ floración femenina; $\mathrm{ASI}=$ intervalo antesis-emergencia de estigmas; $\mathrm{AP}=$ altura de planta; $\mathrm{AM}=$ altura de mazorca; $\mathrm{HM}=$ hileras por mazorca; $\mathrm{LM}=$ longitud de mazorca; $\mathrm{DM}=$ diámetro de mazorca; $\mathrm{P} 100 \mathrm{~S}=$ peso de 100 semillas; $\mathrm{RG}=$ rendimiento de grano; $\mathrm{CV}=$ coeficiente de variación .

La presencia de interacción genotipo x ambiente ( $\mathrm{L} \times \mathrm{Amb})$ en todas las variables estudiadas, demostró que los ambientes de evaluación afectaron de manera importante la expresión fenotípica de las líneas; en general se observó que las líneas con relativo alto rendimiento de grano en el ambiente de riego no fueron las más sobresalientes en el ambiente de sequía. Debido a la presencia de interacción genotipo $\mathrm{x}$ ambiente, la evaluación de genotipos para identificar tolerantes a la sequía, requiere de evaluaciones del rendimiento de grano en condiciones hídricas favorables y desfavorables (Rosielle y Hamblin, 1981), se debe a que los genotipos responden diferencialmente a la condición de humedad y al grado de intensidad de la sequía (Golbashy et al., 2010).

Considerando al rendimiento de grano en sequía, como criterio para elegir líneas contrastantes en respuesta a la tensión hídrica, el Cuadro 2 muestra los promedios de características críticas en los ambientes de sequía y riego de seis líneas de alto y seis de bajo rendimiento relativo bajo sequía. El grupo de líneas sobresalientes en rendimiento en el ambiente de sequía, con respecto al ambiente de riego mostró una reducción de $65.1 \%$ en el rendimiento; sin embargo, la reducción fue mayor (90\%) para el grupo de líneas con bajo rendimiento de grano en sequía. En lo que respecta a los días a floración femenina (FF) tanto las líneas de mayor como las de menor rendimiento de grano en riego, fueron más precoces ( 20 y 24 días respectivamente) en comparación al ambiente de sequía. 
Cuadro 2. Medias de las líneas de mayor y menor rendimiento de grano seleccionadas con base en el rendimiento bajo sequía del grupo de líneas segregantes de maíz (Zea mays L.).

\begin{tabular}{|c|c|c|c|c|c|c|c|c|}
\hline \multirow{2}{*}{ Línea } & \multicolumn{4}{|c|}{ Sequía } & \multicolumn{4}{|c|}{ Riego } \\
\hline & $\mathrm{RG}\left(\mathrm{t} \mathrm{ha}^{-1}\right)$ & FF (d) & ASI (d) & $\mathrm{AP}(\mathrm{cm})$ & $\mathrm{RG}\left(\mathrm{t} \mathrm{ha}^{-1}\right)$ & $\mathrm{FF}(\mathrm{d})$ & ASI (d) & $\mathrm{AP}(\mathrm{cm})$ \\
\hline LUM69 & 2.12 & 84 & 3 & 101 & 2.66 & 65 & 2 & 241 \\
\hline LUM80 & 1.53 & 83 & 3 & 131 & 5.59 & 63 & 1 & 273 \\
\hline LUM57 & 1.23 & 85 & 3 & 123 & 4.02 & 66 & 2 & 252 \\
\hline LUM137 & 1.23 & 82 & 4 & 97 & 3.2 & 60 & 2 & 224 \\
\hline LUM51 & 1.19 & 83 & 3 & 115 & 3.71 & 63 & 0 & 229 \\
\hline LUM188 & 1.15 & 85 & 4 & 130 & 5.05 & 65 & 0 & 278 \\
\hline Media & 1.41 & 84 & 3 & 116 & 4.04 & 64 & 1 & 250 \\
\hline LUM144 & 0.32 & 85 & 3 & 122 & 2.69 & 62 & 2 & 254 \\
\hline LUM134 & 0.3 & 92 & 7 & 128 & 3.93 & 67 & 2 & 271 \\
\hline LUM113 & 0.29 & 92 & 8 & 104 & 1.84 & 65 & 1 & 256 \\
\hline LUM29 & 0.28 & 87 & 6 & 103 & 2.1 & 63 & 2 & 231 \\
\hline LUM108 & 0.27 & 86 & 4 & 109 & 3.21 & 63 & 2 & 243 \\
\hline LUM126 & 0.17 & 93 & 6 & 101 & 2.32 & 67 & 1 & 253 \\
\hline Media & 0.27 & 89 & 6 & 111 & 2.68 & 65 & 1 & 251 \\
\hline $\operatorname{DMS}_{(0.05)}$ & 0.43 & 2.11 & 1.9 & 14.8 & 1.16 & 2.08 & 1 & 10.5 \\
\hline
\end{tabular}

DMS= diferencia mínima significativa.

Para la variable de intervalo antesis emergencia de estigmas, el grupo de líneas sobresalientes en el ambiente de sequía manifestó un retraso de dos días con respecto a las de riego, en tanto que la respuesta en líneas de menor rendimiento, el retraso fue de cuatro días. En lo que concierne a la altura de planta el grupo de líneas sobresalientes mostró una reducción en altura de 53.6\%, con respecto al ambiente de riego y grupo de menor rendimiento reveló una reducción en altura del orden de 55.8. Estos resultados demuestran el efecto la disponibilidad del agua en el comportamiento agronómico de los genotipos, lo que apoya la idea de que las evaluaciones para seleccionar genotipos tolerantes a sequía deben realizarse en ambientes que contrasten de humedad (Messina et al., 2015).

Los análisis de varianza de los índices de susceptibilidad a la sequía (ISS) e índice de tolerancia (IT), permitieron determinar diferencias altamente significativas entre genotipos para ambos índices (Cuadro 3), los coeficientes de variación para los dos índices mostraron valores aceptables de 17.27 y $19.98 \%$, para ISS y IT, respectivamente. Los resultados obtenidos en ambos índices, permite inferir que la población segregante evaluada en esta investigación, posee una gran variabilidad genética en el rendimiento de grano, que se manifiesta tanto en ambientes de riego como en sequía; lo que se reflejó en la gran variación de valores de los índices ISS e IT en los genotipos probados. Se observó, que las líneas con un mayor ISS e IT tuvieron un menor rendimiento de grano en el ambiente de sequía, lo que significa una respuesta de susceptibilidad a la escasez de agua, en tanto que las líneas mostrando valores pequeños en los índices pueden ser clasificadas como tolerantes a la sequía (Estrada et al., 2016). 
Cuadro 3. Cuadrados medios del análisis de varianza de una vía para los índices de susceptibilidad a la sequía (ISS) e índice de tolerancia (IT).

\begin{tabular}{cccc}
\hline FV & GL & ISS & IT \\
\hline Líneas & 99 & $0.103^{* *}$ & $0.486^{* *}$ \\
Error & 300 & 0.027 & 0.09 \\
CV $(\%)$ & & 17.27 & 19.98 \\
\hline
\end{tabular}

$\mathrm{FV}=$ fuente de variación; $\mathrm{GL}=$ grados de libertad; ${ }^{* *}=$ significativo al $1 \% ; \mathrm{CV}=$ coeficiente de variación.

Los índices de susceptibilidad (ISS) y de tolerancia (IT) a la sequía identificaron dos grupos de líneas; uno clasificado como tolerante, compuesto por 13 líneas y un segundo grupo considerado como susceptible, conformado por cinco líneas (Cuadro 4). ambos índices identificaron al mismo grupo de líneas; sin embargo, la posición de las líneas fue distinta. Las líneas clasificadas por los índices como tolerantes, presentaron una reducción en el rendimiento de grano promedio de alrededor de $58.6 \%$ en el ambiente de sequía, mientras que las líneas clasificadas como susceptibles, mostraron una mayor disminución del rendimiento de grano en sequía (88.9\%), estos resultados son similares a los reportados por otros estudios donde las pérdidas de rendimiento en el ambiente de sequía fueron de alrededor de $71.5 \%$, en relación a riego (Golbashy et al., 2010).

Cuadro 4. Líneas tolerantes y susceptibles seleccionadas por el índice de susceptibilidad a la sequía (ISS) y el índice de tolerancia (IT), en la población segregante de maíz (Zea mays L.) evaluada.

\begin{tabular}{ccccc}
\hline Línea & ISS & IT & Sequía RG $\left(\mathrm{t} \mathrm{ha}^{-1}\right)$ & Riego RG $\left(\mathrm{t} \mathrm{ha}^{-1}\right)$ \\
\hline & & & Líneas tolerantes & \\
LUM69 & 0.25 & 0.54 & 2.12 & 2.66 \\
LUM82 & 0.51 & 0.5 & 0.66 & 1.15 \\
LUM30 & 0.53 & 0.46 & 0.6 & 1.06 \\
LUM28 & 0.63 & 0.98 & 0.49 & 1.46 \\
LUM109 & 0.68 & 0.87 & 0.57 & 1.43 \\
LUM10 & 0.69 & 0.83 & 0.69 & 1.51 \\
LUM70 & 0.71 & 1.1 & 0.82 & 1.92 \\
LUM96 & 0.71 & 0.92 & 0.56 & 1.48 \\
LUM153 & 0.72 & 0.95 & 0.61 & 1.56 \\
LUM78 & 0.75 & 1.44 & 0.98 & 2.41 \\
LUM33 & 0.76 & 1.01 & 0.74 & 1.95 \\
LUM101 & 0.8 & 1.03 & 0.52 & 1.35 \\
LUM38 & 0.8 & 0.88 & 0.47 & 1.81 \\
Media & 0.66 & 0.89 & 0.76 &
\end{tabular}




\begin{tabular}{|c|c|c|c|c|}
\hline Línea & ISS & IT & Sequía RG $\left(\mathrm{t} \mathrm{ha}^{-1}\right)$ & Riego RG (t ha-1) \\
\hline \multicolumn{5}{|c|}{ Líneas susceptibles } \\
\hline LUM173 & 1.11 & 3.85 & 0.62 & 4.48 \\
\hline LUM73 & 1.13 & 3.8 & 0.48 & 4.29 \\
\hline LUM97 & 1.13 & 3.4 & 0.47 & 3.87 \\
\hline LUM44 & 1.15 & 3.29 & 0.36 & 3.66 \\
\hline LUM134 & 1.19 & 3.63 & 0.3 & 3.93 \\
\hline Media & 1.14 & 3.59 & 0.45 & 4.05 \\
\hline $\operatorname{DMS}_{(0.05)}$ & 0.23 & 1.23 & 0.43 & 1.16 \\
\hline
\end{tabular}

$\mathrm{DMS}=$ diferencia mínima significativa; $\mathrm{RG}=$ rendimento de grano.

Por otro lado, se identificó a la línea LUM69 con el valor más bajo en el índice ISS (0.25), lo que significa que puede ser clasificada como la línea más tolerante a la sequía, inclusive superó en tolerancia a la línea testigo tolerante T-43 (0.38), adicionalmente la misma línea LUM69 mostró el mayor rendimiento de grano en el ambiente de sequía $\left(2.12 \mathrm{t} \mathrm{ha}^{-1}\right)$; no obstante, en riego no fue una de las de mayor rendimiento. En cuanto al índice IT la línea LUM69 fue una de las tres líneas con mayor tolerancia a sequía con un valor en dicho índice de 0.54 y aunque fue superada por las líneas LUM30 (0.46) y LUM82 (0.5) no hubo diferencia estadística significativa entre las tres líneas.

En cuanto al grupo de líneas clasificadas como susceptibles, las cinco líneas revelaron tener un valor superior a 1.01 en el ISS, valor correspondiente a la línea testigo susceptible Ac7729; sin embargo, no se observaron diferencias estadísticas significativas entre la línea testigo y el grupo de líneas clasificadas como susceptibles. Un similar comportamiento se observó en el índice IT con el grupo de líneas seleccionadas como susceptibles, estas manifestaron valores estadísticamente iguales al de la línea testigo Ac7729 (4.64). Aunque los índices ISS e IT son un criterio aceptable por fitomejoradores para identificar genotipos con tolerancia a sequía, dichos genotipos no necesariamente presentan los mayores rendimientos en el ambiente de riego (Jafari et al., 2009).

Lo anterior se debe a que el rendimiento en riego y sequía no se determinan por las mismas características de la planta, otra causa es que la interacción genotipo x ambiente se vuelve significativa cuando se incrementa el nivel de estrés, adicionalmente cuando se seleccionan genotipos en ambientes no limitantes, se pierde variabilidad genética para tolerancia a algún estrés (Bänziger et al., 2012).

La relación entre los índices ISS e IT con el rendimiento de grano en sequía, generó una pendiente negativa $\left(b=-0.23^{* *}\right)$ entre el ISS y el rendimiento de grano en sequía (Figura 1A); en tanto que, con el rendimiento en riego, la pendiente fue positiva $\left(b=0.08^{* *}\right)$ [Figura $1 \mathrm{~B}$ ]. En cuanto al índice de IT, no se detectó relación con el rendimiento de grano en sequía ( $b=0.61 \mathrm{~ns})$ [Figura 1C]; pero si se observó una marcada relación positiva con el rendimiento de grano en riego $\left(b=0.83^{* *}\right)$ [Figura 1D], resultado similar al encontrado en el ISS, aunque con un mayor ajuste a la recta de regresión. La línea LUM69 identificada como tolerante a la sequía, superó en el valor de ambos índices a la línea testigo tolerante (T-43), se observó en las gráficas como dicha línea se separa del grupo de las líneas tolerantes. 

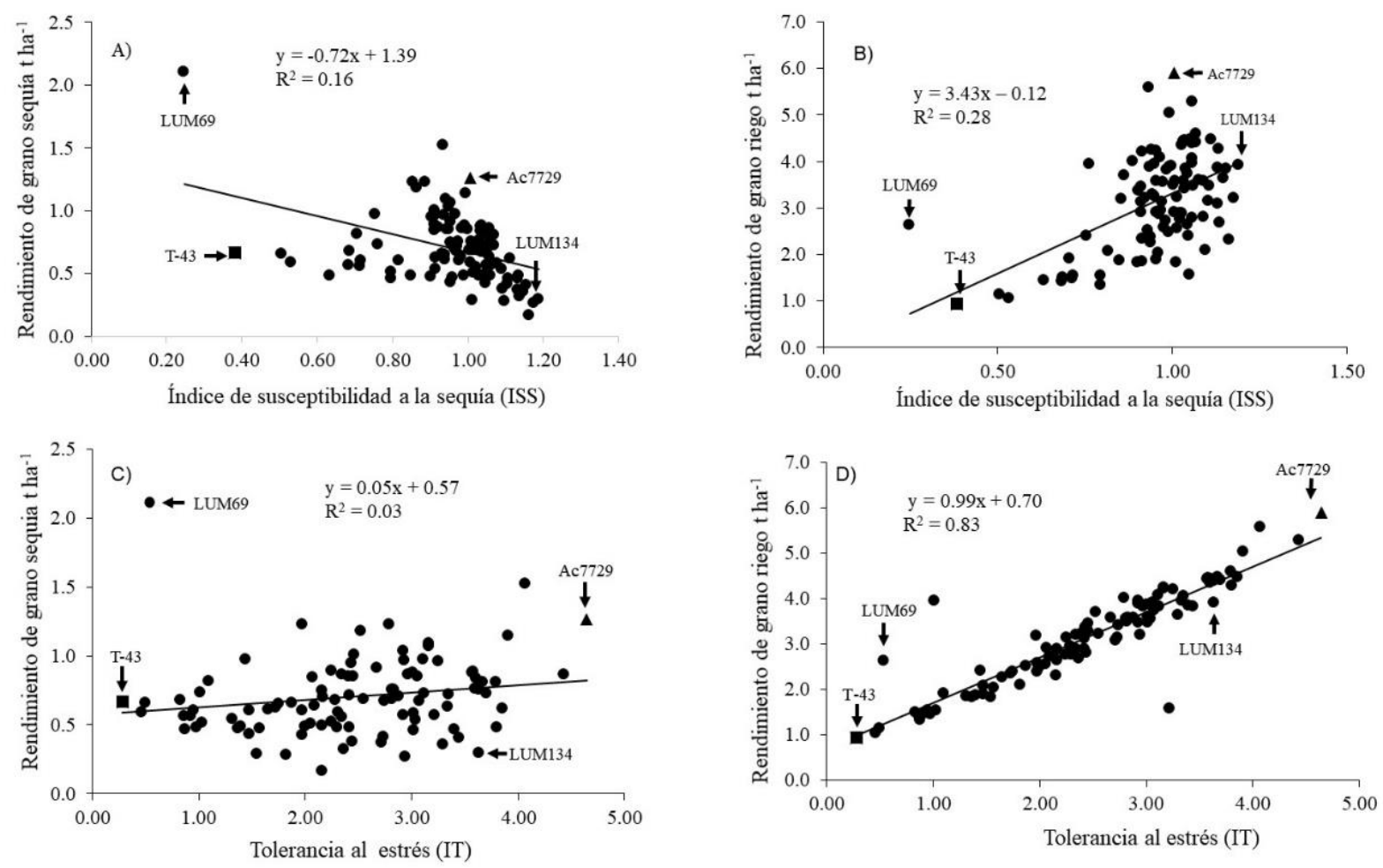

Figura 1. Regresión lineal entre el ISS con el RG bajo condiciones de sequía (A) y de riego (B); y el IT con el rendimiento en sequía (C) y en condiciones de riego (D).

La respuesta de las líneas testigo T-43 (tolerante) y Ac7729 (susceptible) fue la esperada, estas líneas difieren marcadamente en el rendimiento de grano bajo condiciones de riego (5.91 y $0.95 \mathrm{t}$ $\mathrm{ha}^{-1}$, para Ac7729 y T-43, respectivamente), respecto a la reducción en el rendimiento de grano por causa de sequía, la disminución fue muy drástica en la línea Ac7729 (78.7\%), en contraste la disminución en la testigo tolerante (T-43) fue de 29.5\%, el comportamiento de la línea Ac7729 en éste estudio, fue muy similar a lo reportado por Ribaut et al. (1997).

La amplia variación genética presente en el grupo de líneas segregantes y observada en la respuesta en las diez características estudiadas en el ambiente de sequía; puede ser una de las causas principales de la falta de ajuste a la recta de regresión, particularmente con el rendimiento de grano en sequía, se identificó a la línea LUM69 como la de menor reducción del rendimiento en sequía (20.3\%), en contraste LUM134 tuvo la mayor reducción del rendimiento de grano (90.9\%). Los genotipos que presentan bajos valores a través de los índices de ISS y el IT identifican genotipos con buen rendimiento bajo estrés, sin embargo, también son aquellos genotipos que presentan poca diferencia entre el ambiente de riego y sequía (Mohammadi, 2016).

Con base a que los genotipos tolerantes identificados por los índices de ISS e IT, no son los más rendidores bajo condiciones de riego, se obtuvo el promedio a través de ambos ambientes de evaluación (RG combinado), con el propósito de identificar qué líneas integraban grupos contrastantes extremas en RG. En el Cuadro 5 se presentan los dos grupos contrastantes identificados, uno de los resultados determinó que la media del grupo de líneas con mayor RG combinado superó en $69.5 \%$, al promedio del grupo de líneas de bajo RG combinado. 
Cuadro 5. Líneas segregantes de maíz (Zea mays L.) de mayor y menor rendimiento de grano identificadas a través de los ambientes de riego y sequía.

\begin{tabular}{|c|c|c|c|}
\hline Línea & Medias ambos ambientes $\left(\mathrm{t} \mathrm{ha}^{-1}\right)$ & Riego (t ha $\left.{ }^{-1}\right)$ & Sequía $\left(\mathrm{t} \mathrm{ha}^{-1}\right)$ \\
\hline & \multicolumn{3}{|c|}{ Mayor rendimiento } \\
\hline LUM80 & 3.56 & 5.59 & 1.53 \\
\hline LUM188 & 3.1 & 5.05 & 1.15 \\
\hline LUM145 & 3.08 & 5.29 & 0.86 \\
\hline LUM184 & 2.71 & 4.61 & 0.81 \\
\hline LUM22 & 2.68 & 4.47 & 0.89 \\
\hline \multirow[t]{2}{*}{ Media } & 3.02 & 5 & 1.05 \\
\hline & \multicolumn{3}{|c|}{ Menor rendimiento } \\
\hline LUM109 & 1 & 1.433 & 0.57 \\
\hline LUM28 & 0.97 & 1.46 & 0.49 \\
\hline LUM38 & 0.91 & 1.345 & 0.47 \\
\hline LUM82 & 0.9 & 1.154 & 0.66 \\
\hline LUM30 & 0.83 & 1.057 & 0.6 \\
\hline Media & 0.92 & 1.29 & 0.56 \\
\hline $\operatorname{DMS}_{(0.05)}$ & 0.62 & 1.16 & 0.43 \\
\hline
\end{tabular}

$\mathrm{DMS}=$ diferencia mínima significativa.

Al analizar el RG en riego entre ambos grupos contrastantes, se encontró que el grupo de líneas sobresalientes en el RG combinado, tuvieron un RG promedio en riego de $5 \mathrm{t} \mathrm{ha}^{-1}$, superando en $74.2 \%$ al RG de las líneas de bajo RG combinado, en tanto que la misma comparación, pero considerando el RG en sequía; el grupo de líneas de alto RG combinado superó en $37.1 \%$ al rendimiento de grano de las líneas de bajo RG combinado.

En cuanto al grupo de líneas sobresalientes en RG combinado, dichas líneas identificadas no coinciden con las líneas seleccionadas como tolerantes por los índices ISS e IT. Con base a, que no en todos los años se presenta sequía, se debería considerar analizar el comportamiento promedio de genotipos a través de ambientes de humedad, para elegir el germoplasma de mayor tolerancia a la sequía (Rosielle y Hamblin, 1981), debido a que la selección del germoplasma tolerante basada en los índices ISS e IT, identifican genotipos que presentan una menor reducción del RG en condiciones de sequía, pero no garantiza que sean los mejores en condiciones no limitantes de humedad (López et al., 2008).

\section{Conclusiones}

La evaluación de la población segregante de maíz en condiciones de riego y sequía, reveló una gran cantidad de variabilidad genética entre líneas. La intensidad aplicada del déficit hídrico, fue suficiente para inducir la expresión genética de la población e identificar líneas tolerantes a éste factor de estrés, así como producir una reducción en el rendimiento de 77.7\%. Los índices de susceptibilidad a la sequía (ISS) y de tolerancia (IT) clasificaron a las líneas LUM69, LUM82 y LUM30 como las de mayor tolerancia a la sequía, sin embargo, solo la línea LUM69 mostró 
rendimientos competitivos tanto en riego como en sequía. Con base a el promedio a través de ambientes de humedad, las líneas LUM80 (3.56 t ha-1) y LUM188 (3.1 $\left.\mathrm{t} \mathrm{ha}^{-1}\right)$ fueron las de mejor comportamiento. Los índices de ISS e IT aunque son un criterio aceptable para identificar genotipos tolerantes a la sequía, no fueron los mejores debido a que no seleccionan los genotipos con mejor rendimiento de grano bajo condiciones de riego, por lo que también se debe de considerar el promedio del rendimiento de grano de ambientes limitantes y no limitantes de humedad con el fin seleccionar los mejores genotipos con tolerancia a sequía.

\section{Literatura citada}

Agrama, H. A. S. and Moussa, M. E. 1996. Mapping QTLs in breeding for drought tolerance in maize (Zea mays L.). Euphytica. 1(91):89-97.

Avendaño, A. C. H.; Trejo, L. C.; López, C. C.; Molina, G. J. D.; Santacruz, V. A. y Castillo, G. F. 2005. Comparación de la tolerancia a la sequía de cuatro variedades de maíz (Zea mays) y su relación con la acumulación de prolina. Venezuela. Interciencia. 9(30):560-564.

Badu-Apraku, B.; Akinwale, R. O.; Ajala, S. O.; Menkir, A.; Fakorede, M. A. B. and Oyekunle, M. 2011. Relationships among traits of tropical early maize cultivars in contrasting environments. Agron. J. 3(103):717-729. Doi:10.2134/agronj2010.0484.

Bänziger, M.; Edmeades, G. O.; Beck, D. y Bellon, M. 2012. Mejoramiento para aumentar la tolerancia a sequía y a deficiencia de nitrógeno en el maíz: de la teoría a la práctica. Centro Internacional de Mejoramiento de Maíz y Trigo (CIMMYT). México, DF. 68 p.

Bolaños, J. and Edmeades, G. O. 1993. Eight cycles of selection for drought tolerance in lowland tropical Maize. I. Responses in grain yield, biomass, and radiation utilization. Field Crops Res. 3-4(31):233-252. Doi.org/10.1016/0378-4290(93)90064-T.

Bruce, W. B.; Edmeades, G. O. and Barker, T. C. 2002. Molecular and physiological approaches to maize improvement for drought tolerance. J. Exp. Bot. 366(53):13-25. doi.org/10.1093/jexbot/53.366.13.

Castillo-Gutierrez, A. 2004. Drought tolerance and genetic diversity among tropical maize inbred lines. Doctoral Dissertation. University of Nebraska-Lincoln. Lincoln, NE, USA. 112 p.

CONAZA. 2010. Comisión Nacional de Zonas Áridas. Unidad de sequía, desertificación y proyectos especiales. Boletín divulgativo núm. 2. www.conaza.gob.mx.

Edmeades, G. O.; Bolaños, J; Chapman, S. C.; Lafitte, H. R. and Bäzinger, M. 1999. Selection improves drought tolerance in tropical maize populations: I. Gains in biomass, grain yield, and harvest index. Crop Sci. 5(39):1306-1315.

Esparza, M. 2014. La sequía y escasez de agua en México. Situación actual y perspectivas futuras. Secuencia. 89:195-219.

Estrada, P. W.; Jerez M. E.; Nápoles, G. M. C.; Sosa, R. A.; Maceo, R. Y. C. y Cordoví, D. C. 2016. Respuesta de cultivares de frijol (Phaseolus vulgaris L.) a la sequía utilizando diferentes índices de selección. Cultivos Tropicales. 3(37):79-84. Doi.org/10.13140/RG.2.1.5181.2082.

Fischer, K. S.; Edmeades, G. O. and Johnson, E. C. 1989. Selection for the improvement of maize yield under moisture-deficits. Field Crops Res. 3(22):227-243. Doi.org/10.1016/03784290(89)90094-4.

Fischer, R. A. and Maurer, R. 1978. Drought resistance in spring wheat cultivars. Grain yield responses. Aust. J. Agric. Res. 29(5):897-912. Doi.org/10.1071/AR9780897. 
Golbashy, M.; Ebrahimi, M.; Khavari, K. S. and Choukan, R. 2010. Evaluation of drought tolerance of some corn (Zea mays L.) hybrids in Iran. Afr. J. Agric. Res. 19(5):2714-2719.

INEGI. 2008. Instituto Nacional de Estadística Geográfica e Informática. Anuario Estadístico de Morelos. $1^{\mathrm{a}}$ (Ed.). Editorial. México, DF. Tomo II. 461 p.

INIFAP. 2017. Instituto Nacional de Investigaciones Forestales, Agrícolas y Pecuarias (INIFAP). Reporte estación. http://clima.inifap.gob.mx/.

López, S. E.; Tosquy, V. O. H.; Ugalde, A. F. J. y Acosta, G. J. A. 2008. Rendimiento y tolerancia a sequía de genotipos de frijol negro en el estado de Veracruz. Rev. Fitotec. Mex. Núm. Esp. 3(31):35-39.

Khalili, M.; Reza, N. M.; Pour, A. A. and Naseri, R. H. 2013. Effects of drought stress on yield and yield components in maize cultivars (Zea mays L.). Intl. J. Agron. Plant. Prod. 4(4):809-812.

Messina, D. C.; Sinclair, R. T.; Hammer, L. G.; Curan, D.; Thompson, J.; Oler, Z.; Gho, C. and Cooper, M. 2015. Limited-transpiration trait may increase maize drought tolerance in the US corn belt. Agron. J. 6(107):1978-1986. doi:10.2134/agronj15.0016.

Mohammadi, R. 2016. Efficiency of yield-based drought tolerance indices to identify tolerant genotypes in durum wheat. Euphytica. 1(211):71-89.

FAO-SAGARPA. 2012. Organización de las Naciones Unidas para la Alimentación y la Agricultura-Secretaría de Agricultura Ganadería, Desarrollo Rural Pesca y Alimentación. México: el sector agropecuario ante el desafío del cambio climático. México, DF. 439 p.

Ribaut, J. M.; Hoisington, D. A.; Deutsch, J. A.; Jiang, C. and Gonzalez-de-Leon, D. 1996. Identification of quantitative trait loci under drought conditions in tropical maize. 1. Flowering parameters and the anthesis-silking interval. Theor. Appl. Genet. 7(92):887-896.

Ribaut, J. M.; Jiang, C.; Gonzalez, d L. D.; Edmeades, G. O. and Hoisington, D. A. 1997. Identification of quantitative trait loci under drought conditions in tropical maize. 2. Yield components and marker-assisted selection strategies. Theor. Appl. Genet. 7(94):887-896.

Rosielle, A. A. and. Hamblin, J. 1981. Theoretical aspects of selection for yield in stress and nonstress Environments. Crop 6cience. 6(21):943-946. Doi:10.2135/cropsci1981.0011183X002100060033x.

Saini, H. S. and Westgate, M. E. 2000. Reproductive development in grain crops during drought. In Advances in Agronomy. ed. D. L. Spartes. Academic Press. San Diego, CA, USA. 59-96 pp. doi.org/10.1016/S0065-2113(08)60843-3.

Salinas, Z. C. A.; Lluch, B. D.; Hernández, V. S. y Lluch, C. D. B. 1998. La aridez en el noreste de México. Un análisis de variabilidad espacial y temporal. Atmósfera. 1(11):29-44.

SAS. 2009. Statistical Analysis System Institute. SAS user's guide. Statistics. Versión 8.2. SAS Inst. Cary, N. C.

SIAP-SAGARPA. 2017. Servicio de Información Agroalimentaria y Pesquera. Cierre de la producción agrícola por estado SIAP-SAGARPA. http://www.nube.siap.gob.mx/cierre_ agricola.

SMN. 2017. Servicio Meteorológico Nacional. Monitor de sequía en México. http://smn.cna.gob.mx/es/climatologia/monitor-de-sequia/monitor-de-sequia-en-mexico.

Shafiq, S.; Akram, N. A. and Ashraf, M. 2015. Does exogenously-applied trehalose alter oxidative defense system in the edible part of radish (Raphanus sativus L.) under water deficit conditions?. Scientia Horticulturae. 185:68-75. Doi.org/10.1016/j.scienta.2015.01.010. 
Stone, P. J.; Wilson, D. R. and Reid, J. B. 2001. Water deficit effects on sweet corn. I. water use, radiation use efficiency, growth, and yield. Aust. J Agric Res. 52(1):103-113. Doi.org/10.1071/AR99146.

Trujillo, C. A. 2002. Guía para cultivar maíz bajo condiciones de riego en el estado de Morelos. Instituto Nacional de Investigaciones Forestales, Agrícolas y Pecuarias (INIFAP)-Centro de Investigación Regional del Centro Campo Experimental Zacatepec. 19 p.

Walsh, B. 2001. Quantitative genetic. Encyclopedia of life sciences. Nature Publishing Group. 1-7 pp.

Westgate, M. E. and Boyer, J. S. 1986. Reproduction at low silk and pollen water potentials in maize. Crop Sci. 5(26):951-956. Doi:10.2135/cropsci1986.0011183X002600050023x.

Zambrano, Z. E. E.; Limongi, A. J. R.; Alarcón, C. F. D.; Villavicencio, L. J. P.; Caicedo, V. M. B.; Eguez, M. J. F. y Zambrano M. J. J. 2017. Interacción genotipo de híbridos de maíz bajo temporal en Manabí y los Ríos, Ecuador. Espamciencia. 1(8):7-14. 